\title{
Subcellular Targets for Photodynamic Therapy: Implications for Initiation of Apoptosis and Autophagy
}

\author{
Presented by David Kessel, PhD, Wayne State University School of Medicine, Detroit, Michigan
}

\begin{abstract}
Direct lethal effects of photodynamic therapy (PDT) on a cell population were initially shown to occur via initiation of apoptosis, with high doses having a necrotic effect. Selective induction of cellular "self-digestion," better known as autophagy, represents a novel therapeutic target for the prevention of tumor growth and metastasis. It should be noted that autophagy has conflicting roles in the regulation of cell death, which, when applied to oncology, may produce both positive and negative effects on tumor development. Through better understanding the complex parts played by autophagy among diverse cellular signaling pathways in preclinical models, it may be possible to selectively regulate autophagy in response to specific stimuli, thereby inhibiting its oncogenic tendencies while preserving its tumor-suppressive capabilities. (JNCCN 2012;10[Suppl 2]:S56-S59)
\end{abstract}

"In cancer, if you knock out one pathway, two others are there waiting to take over. With photodynamic therapy [PDT], you are knocking out pathways that cannot be replaced," declared David Kessel, PhD, Professor, Department of Pharmacology, Wayne State University School of Medicine, Detroit, Michigan. With more than 50 years of continuous research support from the National Institutes of Health to better understand the mechanisms and potential clinical applications of light-activated therapy, Dr. Kessel focuses here on a

Presented by David Kessel, PhD, Professor, Department of Pharmacology, Wayne State University School of Medicine, Detroit, Michigan.

Dr. Kessel receives funding from the National Institutes of Health for the study of light-activated cancer therapy.

Correspondence: David Kessel, PhD, Department of Pharmacology, Wayne State University School of Medicine, 540 East Canfield Street, Detroit, MI 48201. E-mail: dhkessel@med.wayne.edu behind-the-scenes look at the subcellular environment after PDT. Featured topics of discussion include the mitochondrial and lysosomal pathways to cell death; the cell-protective and cell-destructive effects of autophagy; the good, the bad, and the ugly players in this biochemical arena, particularly singlet oxygen (the bad) and hydroxyl radicals (the ugly); and the photokilling benefits associated with targeting lysosomes.

\section{Pathways to Cell Death From PDT}

A relatively small light dose can cause the death of a significant number of photosensitized tumor cells, explained Dr. Kessel. The death pathways after PDT include the selective loss of $\mathrm{Bcl}-2$, which leads directly to apoptosis and cell death. ${ }^{1}$ However, the protein Bax is left unaffected by PDT, remaining a proapoptotic protein. "After PDT, you get the loss of these proteins that control apoptosis," he added. Perhaps more important is the fact that there is no opportunity to bypass or circumvent these death signals, which Dr. Kessel mentioned is what makes PDT such a successful therapy. "It does not take much to induce cell death. When the signal to initiate apoptosis is provided, eg, via release of cytochrome c from mitochondria, a cell is sent along an irreversible path to cell death."

These cell death mechanisms occur rapidly, said Dr. Kessel. "Within 10, 30, 60 minutes, the cells are becoming apoptotic," he explained. In a preclinical study of mouse lymphoma cells, Agarwal et $\mathrm{al}^{2}$ found that marked degradation of DNA occurred in as little as 30 minutes after PDT-induced photodamage. This genomic DNA fragmentation was dose- and time-dependent, which indicates this response is an enzymatic process related 
to cell killing. ${ }^{2}$ Compared with this mitochondrial pathway to apoptosis after PDT, the lysosomal pathway is a little more indirect, according to Dr. Kessel. "You release lysosomal proteases, and they cleave a protein called BID, leading to cell apoptosis."

\section{Dual Roles of Autophagy: Cell Survival and Cell Death}

In 2006, PDT was found to initiate autophagy, a recycling process that could simply be called cellular "self-digestion." It is a cellular pathway involved in the degradation of proteins and organelles. ${ }^{3}$ According to Dr. Kessel et al, ${ }^{3}$ autophagy was originally characterized as a survival response to nutrient deprivation and other forms of cellular stress.However, studies have shown that the induction of autophagy can sometimes lead to cell death. ${ }^{4-10}$ In addition, autophagic cell death has morphologic and biochemical features that are distinct from both apoptosis and necrosis. ${ }^{3}$ The key cellular actors in the autophagy play are illustrated in Figure 1.

Among its duties, autophagy removes injured organelles and misfolded and aggregated proteins. Persistent damaged proteins may be a source of increased oxidative stress, which can have a detrimental effect on cells. Furthermore, stressed organelles force cells to execute adaptive programs to allow for survival, which ultimately may promote tumor growth. Therefore, insufficient autophagy can no longer provide nutrients, prevent the accumulation of defective proteins and organelles, and manage oxidative stress. Consequently, both intracellular and extracellular events that favor malignant transformation may occur.

Autophagy can also be protective. When PDT induces autophagy, the process can isolate and degrade the photodamaged organelles before they have a chance to release pro-apoptotic signals. Autophagy can thus protect cells from cell death.

In a recent study, Donohue et $\mathrm{al}^{11}$ screened more than 3500 compounds via an automated microscopy assay. They made the surprising observation that the photosensitizer termed benzoporphyrin derivative, and marketed as Verteporfin had the unexpected property of inhibiting autophagy in the dark. "Using this information, we treated cells with high doses of this agent and found that this eliminated the shoulder from the dose-response curve created by the action of autophagy." Thus, "with a high PDT dose and less light, we could see a better photodynamic response because autophagy would be inhibited," revealed Dr. Kessel. "What you can learn from these preliminary studies are clues on how to optimize therapy."

\section{New Photosensitizers: Turning Energy Into Reactive Oxygen Species}

The hallmark of impaired autophagy is the creation of oxidative stress, which results from a disparity between the production and elimination of free radicals and reactive metabolites (reactive oxygen spe-

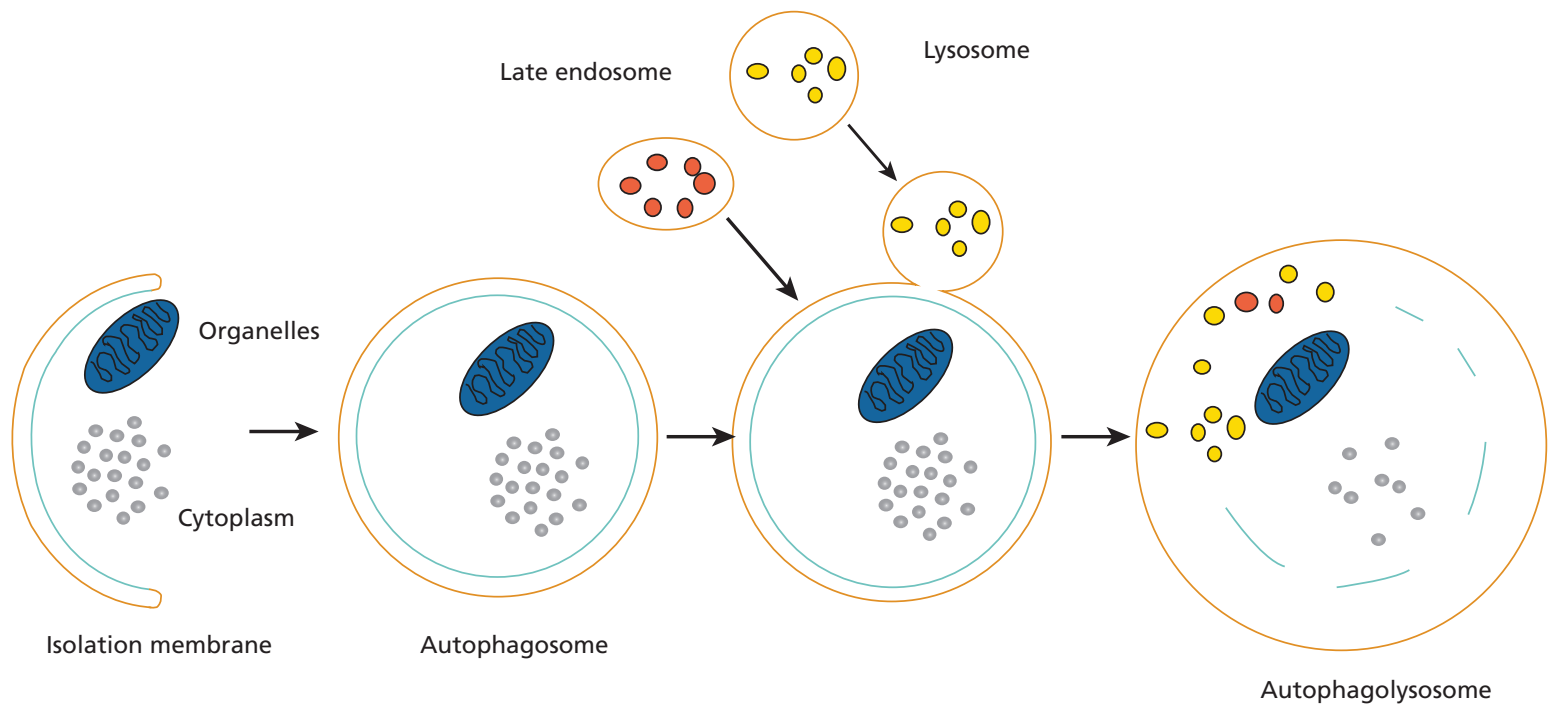

Figure 1 The process of autophagy. 
cies [ROS]). The main source of intracellular ROS is the mitochondrial respiratory chain. All stages of tumor formation, including initiation, promotion, and progression, are impacted by ROS.

The ROS interactions after PDT were briefly discussed by Dr. Kessel. He humorously grouped the players as follows: the good-glutathione, superoxide dismutase (SOD), catalase; the bad-singlet molecular oxygen, superoxide anion radical (hydrogen peroxide); and the ugly-the hydroxyl radical. Although the first ROS formed during irradiation of photosensitized cells is usually singlet molecular oxygen, other ROS have been implicated in the phototoxic effects of PDT, including superoxide anion radical, hydrogen peroxide, and hydroxyl radical. ${ }^{12}$ Price et $\mathrm{al}^{12}$ found that formation of hydrogen peroxide contributes to the efficacy of PDT.

New photosensitizers may lead to a better understanding of ROS and pathways to photokilling, reported Dr. Kessel. Figure 2 shows several types of photosensitizers and their absorbance capacities. For instance, porphyrin has a very weak absorbance of $630 \mathrm{~nm}$, whereas the bacteriochlorins have a very strong absorbance of approximately $750 \mathrm{~nm}$, with the chlorins in the middle between the two (at $660 \mathrm{~nm}$ ), he stated.

Certain types of bacteriopheophorbides are unstable to light (WST1) or are associated with formulation problems (WST9). The photocatalytic generation of oxygen radicals by the water-soluble bacteriopheophorbide WST11 has been studied. ${ }^{6}$ Ashur et $\mathrm{al}^{13}$ showed that in aqueous solution, WST11 generates only superoxide and hydroxyl radicals, with no detectable traces of singlet oxygen. These findings rule out the paradigm

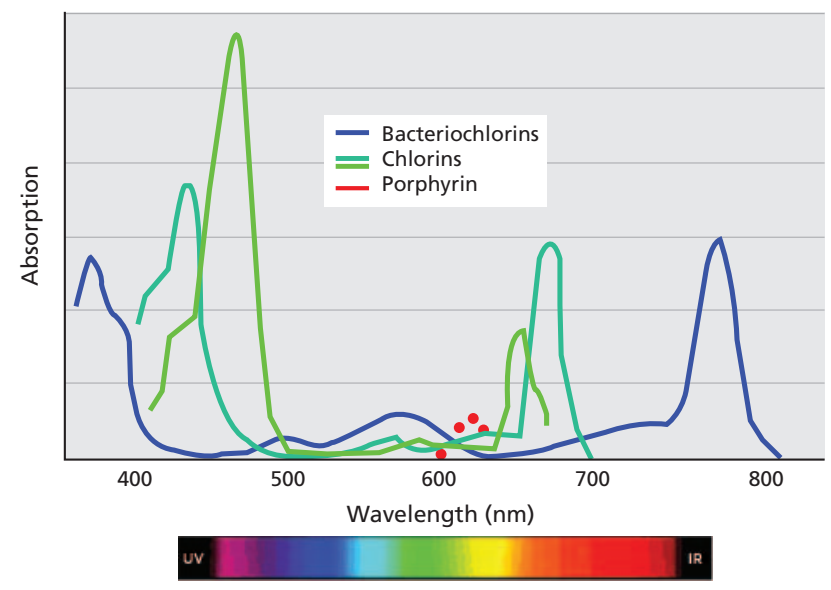

Figure 2 Absorbance of 3 types of photosensitizers. Abbreviations: IR, infared; UV, ultraviolet. that PDT is necessarily mediated by the formation of singlet oxygen. ${ }^{13}$ In addition, WST11 has an extraordinarily weak fluorescence, Dr. Kessel added.

\section{Targeting Lysosomes for More Efficient Photokilling}

Dr. Kessel and colleagues examined 2 photosensitizing agents that target lysosomes: the chlorin NPe6 and the palladium WST11. With both agents, the "shoulder" on the PDT dose-response curve was substantially attenuated, consistent with loss of a cytoprotective pathway. They believe that lysosomal targets may offer the possibility of promoting the efficacy of PDT through eliminating a potentially protective cellular pathway. "These agents are more efficient than porfimer sodium at turning energy into reactive oxygen species, which may be good news," Dr. Kessel pronounced. "We blocked autophagy by lysosomal photodamage, which really is a surprise," he added.

Targeting the mitochondria or the lysosome with PDT may result in a different effect on autophagy, stated Dr. Kessel. For instance, with a mitochondrial target, autophagy is protective with the autophagyrelated proteins $5(A \operatorname{tg} 5)$ and $7(A \operatorname{tg} 7)$, so the efficacy of PDT is enhanced. However, with a lysosomal target, there is no autophagic protection and a nonAtg5 effect, he explained. Preclinical studies have indicated that loss of Atg7 inhibited autophagy and enhanced the cytotoxicity of photosensitizers that mediate photodamage to the mitochondria or the endoplasmic reticulum. Dr. Kessel and colleagues found that a deficiency in Atg7 or Atg5 suppressed the induction of autophagy in PDT protocols with either NPe6 or WST11. They proposed that the efficacy of lysosomal photosensitizers may be partly from both promotion of autophagic stress and suppression of autophagic prosurvival functions.

Selective photodamage can provide information about cell biology, stated Dr. Kessel. To improve the efficacy of PDT, targeting lysosomes should provide more efficient photokilling, because autophagy is prevented, he noted. Rodriguez et $\mathrm{al}^{14}$ determined that the lysosomal sensitizer was effective at a much lower dose than was the mitochondrial sensitizer. "PDT is the only way I can think of to punch holes in the lysosomes," quipped Dr. Kessel. "The take home message is that maybe people will start using PDT for other purposes." 


\section{Conclusions}

Over the years, much has been learned about the conflicting cell-protective and cell-destructive functions of autophagy as a result of the photodynamic effect in preclinical models. Yet much still needs to be learned about how to identify the relevant cellular signaling pathways and to selectively regulate autophagy in response to specific stimuli. It appears that lysosomes may be the optimal target to improve the efficacy of PDT. A better understanding of the subcellular environment may be the ultimate key to targeting autophagy through PDT to prevent tumor growth and metastasis.

\section{References}

1. Kim HR, Luo Y, Li G, Kessel D. Enhanced apoptotic response to photodynamic therapy after bcl-2 transfection. Cancer Res 1999;59:3429-3432.

2. Agarwal ML, Clay ME, Harvey EJ, et al. Photodynamic therapy induces rapid cell death by apoptosis in L5178Y mouse lymphoma cells. Cancer Res 1991;51:5993-5996.

3. Kessel D, Vicente MG, Reiners JJ Jr. Initiation of apoptosis and autophagy by photodynamic therapy. Lasers Surg Med 2006;38:482-488.

4. Gozuacik D, Kimchi A. Autophagy as a cell death and tumor suppressor mechanism. Oncogene 2004;23:2891-2906.
5. Yu L, Alva A, Su H, et al. Regulation of an ATG7- beclin 1 program of autophagic cell death by caspase-8. Science 2004;304:15001502.

6. Edinger AL, Thompson CB. Death by design: apoptosis, necrosis and autophagy. Curr Opin Cell Biol 2004;16:663-669.

7. Eskelinen EL. Doctor Jekyll and Mister Hyde: autophagy can promote both cell survival and cell death. Cell Death Differ 2005;12(Suppl 2):1468-1472.

8. Tsujimoto Y, Shimizu S. Another way to die: autophagic programmed cell death. Cell Death Differ 2005;12(Suppl 2):1528 1534.

9. Lockshin RA, Zakeri Z. Apoptosis, autophagy, and more. Int J Biochem Cell Biol 2004;36:2405-2419.

10. Guillon-Munos A, van Bemmelen MX, Clarke PG. Role of phosphoinositide 3-kinase in the autophagic death of serumdeprived PC12 cells. Apoptosis 2005;10:1031-1041.

11. Donohue E, Tovey A, Vogl AW, et al. Inhibition of autophagosome formation by the benzoporphyrin derivative verteporfin. J Biol Chem 2011;286:7290-7300.

12. Price M, Terlecky SR, Kessel D. A role for hydrogen peroxide in the pro-apoptotic effects of photodynamic therapy. Photochem Photobiol 2009;85:1491-1496.

13. Ashur I, Goldschmidt R, Pinkas I, et al. Photocatalytic generation of oxygen radicals by the water-soluble bacteriochlorophyll derivative WST11, noncovalently bound to serum albumin. J Phys Chem A 2009;113:8027-8037.

14. Rodriguez ME, Zhang P, Azizuddin K, et al. Structural factors and mechanisms underlying the improved photodynamic cell killing with silicon phthalocyanine photosensitizers directed to lysosomes versus mitochondria. Photochem Photobiol 2009;85:1189-1200. 